\title{
Sleep-Disordered Breathing in Michigan: A Practice Pattern Survey
}

\author{
Ronald D. Chervin, M.D., M.S., 1 Cheryl A. Moyer, M.P.H.,2 \\ John Palmisano, M.S., 1 Alon Y. Avidan, M.D., M.P.H., 1 \\ Emerson Robinson, D.D.S., M.P.H., ${ }^{3}$ Susan L. Garetz, M.D., M.S., 4 and \\ Joseph I. Helman, D.M.D.5
}

Objectives: This survey sought to determine whether self-professed sleep specialists in the State of Michigan show practice variations in the diagnosis and management of sleep-disordered breathing (SDB), and whether such variations occur between pulmonologists and neurologists. Methods: Questionnaires on practice volume and patterns during the prior 12 months were mailed to physician members of the Michigan Sleep Disorders Association ( $n=119)$; 67 were completed and returned. Results: Respondents reported that they personally saw a median of 8 new patients each week for suspected SDB; estimates were that $86 \%$ of these patients were eventually confirmed to have SDB. Most patients (82\%) had laboratory-based polysomnography after an initial clinic evaluation, and most (69\%) of those treated for SDB received continuous positive airway pressure. However, practice patterns differed substantially among respondents, even when the analysis was limited to the 42 who reported board certification by the American Board of Sleep Medicine. For example, among all surveyed practices the likelihood that suspected SDB would be evaluated with a split-night diagnostic and treatment polysomnogram varied from 0 to $90 \%$. The likelihood of SDB treatment with bilevel positive airway pressure varied from 0 to $50 \%$, with automatically titrating devices from 0 to $100 \%$, with surgery from 0 to $100 \%$ (0 to $50 \%$ among certified practitioners), and with oral appliances from 0 to $20 \%$. The practice patterns of pulmonologists and neurologists did not differ significantly. Conclusion: Approaches to SDB vary widely in Michigan, though not according to clinician background in pulmo-

Sleep and Breathing, volume 7, number 3, 2003. Address for correspondence and reprint requests: Ronald D. Chervin, M.D., Michael S. Aldrich Sleep Disorders Laboratory, 8D8702 University Hospital, Box 0117, 1500 E. Medical Center Dr., Ann Arbor, MI 48109-0117. Email: chervin@umich.edu. ${ }^{1}$ Sleep Disorders Center, Department of Neurology; ${ }^{2}$ Global REACH (Research, Education and Collaboration in Health); ${ }^{3}$ Department of Prosthodontics, School of Dentistry; ${ }^{4}$ Department of Otolaryngology-Head and Neck Surgery; and ${ }^{5}$ Department of Surgery, Section of Oral and Maxillofacial Surgery; University of Michigan Health System, Ann Arbor, Michigan. Copyright (C) 2003 by Thieme Medical Publishers, Inc., 333 Seventh Avenue, New York, NY 10001, USA. Tel: +1(212) 584-4662. 15209512,p;2003,07,03,095,104,ftx,en;sbr00234x. 
nary medicine or neurology. A patient's experience, in both assessment and treatment, could differ substantially based on which clinician is consulted.

KEYWORDS: Sleep apnea syndromes, physician's practice patterns, polysomnography, continuous positive airway pressure

\begin{abstract}
Cost-effectiveness analyses help to motivate decisions about healthcare expenditures, and in particular could influence the direction that sleep medicine takes in its comparatively early stages of development. However, cost-effectiveness analyses in sleep medicine have been rare. ${ }^{1}$ All such analyses must compare one strategy or procedure-usually a newly proposed intervention- to another method, most often standard practice. Lack of knowledge about current practices constitutes part of the reason why more cost-effectiveness analyses have not been performed. A study by Richard Coleman and colleagues in the early $1980{ }^{\prime}{ }^{2}{ }^{2}$ and a follow-up study more recently, ${ }^{3}$ showed that sleep-disordered breathing (SDB) is by far the most common clinical diagnosis and reason for testing at large academic sleep centers. However, these studies did not examine clinicians' practice patterns with respect to $\mathrm{SDB}$, or the experience of their patients.

In countries that do not have nationalized healthcare systems and records, practice pattern studies still can be accomplished conveniently within specific healthcare systems, health maintenance organizations, or third-party payors. However, more generalizable, regional data are more difficult to obtain. We surveyed physicians who view themselves as sleep specialists, as suggested by membership in the Michigan Sleep Disorders Association, about their experience, practices, diagnostic procedures, and management strategies with respect to SDB. The aim of this descriptive study was to provide estimates of practice patterns with respect to SDB. Such data are illuminating by themselves, and they also facilitate development of cost-effectiveness models.
\end{abstract}

\section{METHODS}

\section{Subjects}

Names and addresses were obtained for all Michigan Sleep Disorders Association members who are physicians. The Michigan Sleep Disorders Association is believed to have been the first state-based organization of its kind at its founding in 1980. The organization sponsors a well-attended annual meeting, a smaller annual research meeting, a newsletter, and a Web site. Although membership in this organization is not required to practice sleep medicine in Michigan, membership is comparatively inexpensive ( $\$ 50$ per year) and the large majority of clinicians with active sleep medicine practices are believed to belong to this organization. The number of clinician members compares closely to the Michigan membership roster listed in the American Academy of Sleep Medicine Directory, 2002$2003(n=159$, including several technicians, research associates, or other nonclinicians). On August 17, 2001, Internal Review Board-approved cover letters and questionnaires were mailed to all 119 physician members of the Michigan Sleep Disorders Association. A lengthy consent that could have reduced response rates was avoided by keeping this IRB-approved survey anonymous. Respondents were asked to mail a coded postcard separately, at the same time that they mailed the survey, to let investigators know their questionnaire had been completed. On September 14, 2001, nonresponders were mailed a follow-up request to complete the survey. All responses included in this report were received by the end of 2001 . 


\section{Table 1 Assessment and Treatment of Sleep-Disordered Breathing: A Practice Pattern Survey}

\section{Thinking about the past 12 months, please give your best estimates to the questions below.}

1) About how many new patients did you evaluate (personally see) for suspected sleep-disordered breathing during an average week:

Average \# of new patients evaluated per week = (If 0, skip to Q8)

o mostly adults

o mostly children

2) After your assessment and any relevant testing or referrals, what percentage of such patients were confirmed to have ...
a) sleep disordered breathing
- $\%$
b) no significant sleep-disordered breathing
— $\%$

3) Among all the new patients you saw for sleep-disordered breathing during the past 12 months, what is your best estimate of the percent who...
a) went on to have a sleep study
— \% Don't know
b) already had a sleep study before they saw you
- $\%$
- Don't know
c) had no sleep study before or after seeing you
$\%$
o Don't know

4) Of those new patients you subsequently assessed with sleep laboratory studies, what percent had a
a) full diagnostic night
- $\%$
o Don't know
b) split-night protocol
o Don't know

5) Among the new patients you saw for possible sleep-disordered breathing, about what percent did you assess with an ambulatory study?

$$
\text { —If 0, skip to Q7) } \% \text { Don't know }
$$

6) Of those you assessed with an ambulatory study, about what percent had an ambulatory study that ...
a) ... included EEG leads
_ \% o Don't know
b) ... included at least 2 cardio-respiratory channels
- $\%$
o Don't know
c) ... used oximetry only
- $\%$
- Don't know
d) ... titrated CPAP automatically at the same time
$\%$
o Don't know
e) Other:
— $\%$
o Don't know

7) During an average week, in how many newly diagnosed patients did you initiate treatment for sleep-disordered breathing? patients per week (If 0, skip to Q15, next page)

8) During an average week, how many patients with sleep-disordered breathing did you see for follow-up visits? patients per week

9) Among all the patients you treated, yourself or by referral, for sleep-disordered breathing, what percent received ... (sum of responses may exceed $100 \%$ ):
a) CPAP
- $\%$
- Don't know
b) BiPAP
- $\%$
- Don't know
c) Automatic self-titrating CPAP
- $\%$
o Don't know
d) Surgery
$\%$
o Don't know
e) Oral appliance
- $\%$
o Don't know
f) A formal weight loss program
- $\%$
o Don't know
g) Positional therapy
— $\%$
o Don't know
h) Other
- $\%$
- Don't know

10) Among all the patients you treated with CPAP or BiPAP, what percent underwent a titration study?

$$
\text { _ \% o Don't know }
$$

11) Among the patients you saw who subsequently had surgery for sleep-disordered breathing, what percent ...
a) had inpatient surgery (any type)
— $\%$
- Don't know
b) had outpatient surgery (any type)
$\%$
- Don't know
c) failed treatment with CPAP or BiPAP
- \%
o Don't know 


\section{Table 1 (Continued)}

12) Of those sleep-disordered breathing patients who had inpatient surgery, what percent had (sum of responses may exceed $100 \%)$...
a) Uvulopalatopharyngoplasty (UPPP)
- $\%$
o Don't know
b) Tracheostomy
- $\%$
o Don't know
c) Tonsillectomy or adenotonsillectomy
- $\%$
o Don't know
d) Genioglossus advancement
- $\%$
o Don't know
e) Hyoid suspension
- $\%$
o Don't know
f) Maxillary and mandibular advancement
o Don't know
g) Other
- \%
o Don't know

13) Of those sleep-disordered breathing patients who had outpatient surgery, what percent were treated with ...
a) Laser-assisted uvuloplasty (LAUP)
b) Radiofrequency volumetric reduction of
- $\%$
o Don't know
the soft palate or tongue (somnoplasty)
o Don't know
c) Tonsillectomy or adenotonsillectomy
- $\%$
o Don't know
d) Other
o Don't know

14) Of those sleep-disordered breathing patients who were treated with oral appliances, what percent received . . .
a) An adjustable mandibular advancement device
- $\%$
o Don't know
b) A nonadjustable mandibular advancement device
- Don't know
c) Tongue retaining device
o Don't know
d) Other
$\%$
$\%$
o Don't know

Now we'd like to ask some basic questions about you.

15) Please indicate which of the following degrees you have:
$\circ \mathrm{MD}$
O DDS
$\circ \mathrm{PhD}$
O MS/MA
o Other;

16) What year did you graduate from medical, graduate, or other professional school?

17) What is your primary clinical specialty?

18) Are you board certified in that specialty?

oYes o No

19) Are you a member of any of the following groups?
a) American Academy of Sleep Medicine (AASM)
OYes ONo
b) Sleep Research Society (SRS)
oYes ONo
c) Academy of Dental Sleep Medicine (ADSM)
OYes ONo
d) State or regional sleep disorders association
oYes o No

20) Which best describes the main way that you obtained your training in sleep medicine? (Please mark one response only) ○ No formal training

o Trained on the job by colleagues

o Took postgraduate course that lasted 2 weeks or less

- Postgraduate courses or fellowship work that amounted to less than 1 year full-time effort

o Postgraduate fellowship work that amounted to 1 or more years of full-time effort

21) Did you obtain certification by the American Board of Sleep Medicine?

oYes o No

22) Which best describes your current practice setting?

o Private practice or free-standing sleep laboratory

o Hospital or HMO staff

o Academic medicine

o Other

23) In what state (or country, if not U.S.) do you see most of your patients?

24) What is your gender? o Male o Female

Thank you for your time. 


\section{Survey Instrument}

We designed a new questionnaire (see Table 1) about physicians' practices, experience, diagnostic approaches, and management strategies during the prior 12 months with respect to SDB. The survey development process included several stages: exploratory interviews, question drafting, and pretesting that allowed revision and finalization. In the first-stage exploratory interviews, open-ended discussions with sleep medicine physicians were used to understand how they framed their experiences with diagnosis and treatment of SBD. In the second stage, question drafting, survey items were generated based on the wording and response options suggested in the open-ended interviews. For example, based on physician preferences, several items requested percentages rather than raw numbers to estimate past experience with patients and procedures. During the third stage, pretesting, early versions of the questionnaire were administered twice, among six sleep medicine specialists, to collect and incorporate feedback about appropriateness of response options, clarity of wording, logic of concepts, and ability to remember and estimate answers. The survey was designed in accordance with Dillman's principles of questionnaire design. ${ }^{4}$ Despite precautions taken and refinement during pilot tests, three items (numbers 11, 13, and 14) proved to generate illogical answers with sufficient frequency to warrant exclusion of collected data from this report. The remainder of the items generated responses that were generally free of obvious inconsistencies, and these data were analyzed further.

\section{Analysis}

All data were double-entered, to improve accuracy, into an electronic database. Responses for most items are best estimates of numbers or percents, with reference to the past 12 months of practice (see Table 1). "Don't know" responses were considered to be missing data. Response variables generally did not follow a normal distribution; therefore nonparamet- ric analytic methods were used. Data were summarized by median, tenth and ninetieth percentile, maximum and minimum. Percentages were compared using Wilcoxon rank sums tests or chi-square tests. In some cases, analyses were repeated after excluding respondents who were not certified by the American Board of Sleep Medicine (ABSM). Data were analyzed to characterize clinician practices, but also to generate an approximate characterization of patient experiences in Michigan practices. The latter was accomplished by weighting responses from each practitioner by the estimated number of patients seen or treated by that practitioner. Analyses were conducted using $\mathrm{SAS}^{\circledR}$, version 8.1 (SAS Institute Inc., Cary, NC). The level of significance was set at $\mathrm{p}<0.05$.

\section{RESULTS}

\section{Respondents}

A total of 77 (65\%) of the mailed surveys were returned. Surveys excluded from analysis included 7 from clinicians who were retired and saw no patients during the last 12 months, 2 from respondents who indicated they were dentists (despite the initial effort to confine the survey to physicians), and 1 from a clinician who answered the first two items only. This left 67 surveys on which the data reported below are based.

Among these 67 respondents, 61 (91\%) were male. An M.D. degree was held by 57 (85\%) of respondents, and a D.O. by 10 (15\%). In addition, 6 (9\%) held a Ph.D., M.S., M.A., M.B.A., or M.P.H. The median year of attainment of professional degree was 1981 (range, 1959 to 1994). The 66 respondents who indicated their specialty included 29 (44\%) pulmonologists, 21 (32\%) neurologists, 6 (9\%) otolaryngologists, 4 (6\%) primary care physicians, 2 (3\%) who listed sleep medicine as their primary specialty, and 1 (1\%) who listed each of the following as a primary specialty: neurophysiology, psychiatry, pediatrics, and anesthesia. Almost all 
( $n=65$, or $97 \%$ ) indicated that they were board certified in their primary specialty, 42 (63\%) were board certified by the ABSM, 63 (94\%) were members of the American Academy of Sleep Medicine, and 14 (21\%) were members of the Sleep Research Society. A total of 16 (24\%) reported that the main way they obtained training in sleep medicine was through postgraduate fellowship work that amounted to 1 or more years of full-time effort; $26(39 \%)$ by postgraduate courses or fellowship work for less than 1 year; 17 (25\%) through on-the-job training by colleagues; 7 (10\%) through postgraduate course work that lasted 2 weeks or less; an additional 6 (9\%) who combined such course work with other methods; and $1(1 \%)$ through none of the above means.

Almost all respondents $(n=58$, or $97 \%$ of those who answered this question) saw most of their patients in Michigan. Most respondents ( $n=45$, $67 \%)$ were in private practice or worked at a freestanding sleep laboratory, whereas $8(12 \%)$ worked as staff at a hospital or other organization, 12 (18\%) worked in academic medicine, 2 (3\%) worked in some other setting, and 2 (3\%) reported combinations of the above.

\section{Practice Patterns}

The number of patients seen annually, derived by multiplication of weekly estimates by 50 , varied by a factor of 40 , as shown in Table 2 . The percentage of newly evaluated patients confirmed to have SDB was usually substantially higher than 50\%. Most had a sleep study after an initial evaluation by a respon- dent, though a minority already had had such a study before the clinic visit. The percentage of $\mathrm{pa}^{-}$ tients evaluated by split-night studies was generally low, but varied widely. Few clinicians reported use of ambulatory studies. Whether or not respondents were ABSM-certified had essentially no effect on median responses and little effect on ranges of responses except for two instances: the minimum number of newly evaluated patients who went on to have a sleep study rose from $10 \%$ among all respondents to $60 \%$ among ABSM-certified respondents, and the maximum number that had already had a sleep study at the time of the initial clinic visit fell from $94 \%$ to $50 \%$.

The number of newly diagnosed patients in whom respondents initiated treatment varied widely, as shown in Table 3 . The large majority treated mainly with continuous positive airway pressure (CPAP), though some outliers were noted, even among ABSM-certified respondents, one of whom treated only $20 \%$ of his or her patients with CPAP. Similarly, wide variability was noted in use of bilevel positive airway pressure (BPAP) and automatically titrating CPAP. Although one practitioner used the latter in $100 \%$ of the patients he or she treated for $\mathrm{SDB}$, the highest percentage among ABSM-certified respondents was 20\%. Most but not all practitioners reported that all their patients underwent a titration study before use of CPAP or BPAP. One ABSM-certified practitioner reported that only $50 \%$ of the patients he or she treated with CPAP or BPAP underwent a titration study first, though the question may have been misunderstood to apply to patients with conditions other than SDB.

Table 2 Practice Patterns: Diagnosis of SDB

\begin{tabular}{lrrrrr}
\hline Question-Item (See Table 1) & $\begin{array}{c}\text { 90th } \\
\text { Max. }\end{array}$ & $\begin{array}{r}\text { 50th } \\
\text { \%ile }\end{array}$ & $\begin{array}{r}\text { 10th } \\
\text { \%ile }\end{array}$ & \%ile & Min. \\
\hline 1. Number new patients seen per year & 2000 & 1000 & 400 & 100 & 50 \\
2. Confirmed to have SDB (\%) & 100 & 95 & 80 & 60 & 50 \\
3a. Went on to have sleep study (\%) & 100 & 96 & 88 & 60 & 10 \\
3b. Already had sleep study before clinic visit (\%) & 94 & 40 & 10 & 0 & 0 \\
4b. Had a split-night study (\%) & 90 & 50 & 13 & 0 & 0 \\
5. Assessed with an ambulatory study (\%) & 10 & 0 & 0 & 0 & 0 \\
\hline
\end{tabular}


Table 3 Practice Patterns: Management of SDB

\begin{tabular}{|c|c|c|c|c|c|}
\hline Question-Item (see Table 1) & Max. & $\begin{array}{l}\text { 90th } \\
\text { \%ile }\end{array}$ & $\begin{array}{l}\text { 50th } \\
\% \text { ile }\end{array}$ & $\begin{array}{l}\text { 10th } \\
\text { \%ile }\end{array}$ & Min. \\
\hline 7. Number patients treated for SDB (in one year) & 2500 & 800 & 300 & 50 & 0 \\
\hline \multicolumn{6}{|l|}{ 9. \% Who received ... . } \\
\hline 9а. . . СРAP & 100 & 90 & 80 & 30 & 0 \\
\hline 9b. . . BPAP & 50 & 20 & 10 & 1 & 0 \\
\hline 9c. . . a automatically titrating CPAP & 100 & 5 & 0 & 0 & 0 \\
\hline $9 \mathrm{~d} . \ldots$ surgery & 100 & 50 & 5 & 0 & 0 \\
\hline 9e. . . oral appliance & 20 & 10 & 1 & 0 & 0 \\
\hline 9f. . . formal weight-loss program & 100 & 65 & 5 & 0 & 0 \\
\hline 9g. . . positional therapy & 100 & 25 & 3 & 0 & 0 \\
\hline 9h. ... other & 20 & 5 & 0 & 0 & 0 \\
\hline $\begin{array}{l}\text { 10. \% of patients treated with CPAP or BPAP } \\
\text { who underwent a titration study }\end{array}$ & 100 & 100 & 100 & 95 & 50 \\
\hline \multicolumn{6}{|l|}{ 12. Among inpatient surgeries, $\%$ who received... } \\
\hline 12a. . . uvulopalatopharyngoplasty & 100 & 100 & 75 & 0 & 0 \\
\hline 12b. ... tracheostomy & 15 & 2 & 0 & 0 & 0 \\
\hline 12c. . . tonsillectomy or adenotonsillectomy & 100 & 90 & 10 & 0 & 0 \\
\hline 12d. . . genioglossus advancement & 30 & 1 & 0 & 0 & 0 \\
\hline 12e. . . . hyoid suspension & 30 & 0 & 0 & 0 & 0 \\
\hline 12f. . . . bimaxillary advancement & 60 & 10 & 0 & 0 & 0 \\
\hline $12 \mathrm{~g} . .$. other & 80 & 1 & 0 & 0 & 0 \\
\hline
\end{tabular}

Surgery and oral appliances were used in widely varying proportions of each practitioner's patients (Table 3), and specifically in 0 to $50 \%$ and 0 to $20 \%$, respectively, among ABSM-certified practitioners. Use of formal weight-loss programs and positional therapy showed wide variability between practitioners. Types of traditional, inpatient surgical procedures varied widely between practitioners. However, large numbers reported that their patients had uvulopalatopharyngoplasty, whereas many fewer reported other types of surgery (Table 3). The ABSM-certified respondents again differed from remaining respondents only in the extremes, for only two procedures. The maximum percentage of inpatient surgeries that were hyoid suspensions was only $1 \%$ among the smaller group of certified practitioners, instead of $30 \%$ in the whole group, and the number of "other" surgeries was 0 in the ABSM group, instead of $80 \%$. In contrast, the maximum percentage of practitioners' patients who received bimaxillary advancement was identical (60\%) among ABSM-certified and noncertified respondents.

Pulmonologists and neurologists, the most common primary specialties represented in the sample, showed no significant differences or trends (Wilcoxon rank sums $\mathrm{p}>0.10$ ) in numbers of $\mathrm{pa}-$ tients evaluated for SDB, rate of confirmation of SDB, tendency for patients to have a sleep study after a clinic evaluation, tendency to have a splitnight study, likelihood of an ambulatory study, numbers of patients treated, type of treatment prescribed, or-for those patients who had surgery-type of surgery performed.

\section{Patient Experience}

The total estimated number of patients evaluated during the past 12 months for suspected SDB, by 65 respondents who answered this question, was 
32,850 . The percentage of these patients who were eventually confirmed to have SDB was $86 \%$ (based on $n=61$ responses). About $82 \%$ (65 responses) went on to have a sleep study after their initial clinical sleep evaluation, whereas $9 \%$ (55 responses) had no sleep study before or after this evaluation. Of those patients subsequently assessed with sleep laboratory studies, 74\% (65 responses) had a full-night diagnostic study, whereas 26\% (65 responses) had a split-night protocol. Only $0.4 \%$ (61 responses) were assessed with ambulatory studies. The total number of patients treated was an estimated 23,500 (by 61 respondents). CPAP was given to $69 \%$ (based on 59 responses), BPAP to $12 \%$ (59 responses), an automatically self-titrating PAP device to $2 \%$ (59 responses), surgery to $12 \%$ (59 responses), oral appliances to $4 \%$ (59 responses), a formal weight-loss program to $18 \%$ (57 responses), positional therapy to $12 \%$ (59 responses), and other treatments to $1 \%$ (58 responses). (These treatments were not mutually exclusive.)

\section{Selection Bias}

Although the investigators could not directly compare data from respondents with those of nonrespondents, it was possible to assess for potential differences by comparison of surveys returned initially to the 13 apparently returned only in response to the second, reminder notice. No significant differences between groups were detected in the numbers or percentages of patients evaluated for suspected SDB, confirmed to have SDB, studied by polysomnography, treated for SDB, seen in followup for SDB, treated with CPAP, treated with BPAP, treated by automatically self-titrating CPAP, treated by surgery, treated with an oral appliance, or titrated before treatment with CPAP or BPAP (Wilcoxon rank sums, $p>0.10$ for each). Backgrounds of the 13 late responders also showed no difference, compared with earlier responders, in percentage of respondents who were pulmonologists versus other specialists, or who held certification by the ABSM. The year of degree completion also showed no difference.

\section{DISCUSSION}

This practice pattern survey, conducted among physician members of a state sleep disorders association, is among the first to characterize frequencies with which physicians who are sleep specialists use different diagnostic and treatment strategies in their approaches to SDB. Most striking is the wide variation, even within one state, in methods of SDB management. This variation persisted when analyses were confined to practitioners board certified by the ABSM. Similarly, background and training experience in sleep medicine also varied widely, though respondents with training in pulmonary medicine and neurology - the two most frequently represented specialties-did not show any consistent differences in their approaches to diagnosis or treatment of SDB. From the perspective of the patient experience in Michigan, current results suggest that $\sim 2$ in 3 treated for SDB received CPAP; 1 in 8 received BPAP; 1 in 8 received surgery; and only 1 in 25 received an oral appliance. Limitations of this study include absence of any data from about one third of the target sample. However, these results provide a rare glimpse of practice patterns that determine resource utilization in the management of a common, treatable disorder with substantial impact on overall health and quality of life.

The field of sleep medicine originally emerged, in large part, because of psychiatrists' and psychologists' interest in relationships between sleep and psychiatric disease. Subsequently neurologists and then pulmonologists became the most frequently represented practitioners in sleep medicine, to an extent highlighted by the current finding that only one psychiatrist was represented in the sample. Several psychologists also belong to the Michigan Sleep 
Disorders Association, but were not included in the current survey. Representation in the sample of primary care physicians ( $6 \%$ of respondents) suggests an encouraging beginning. The epidemiology of $\mathrm{SDB}^{5}$ and the fact that the disease remains undiagnosed in most patients ${ }^{6}$ suggest that adequate control of the condition, from a public health perspective, will only be accomplished by increased interest and involvement of primary care practitioners.

Training in sleep medicine has varied considerably, as reflected in the backgrounds of survey respondents: less than 1 in 4 had had 1 or more years of fellowship training, which is now for the first time required to sit for the ABSM examination. Interestingly, current findings suggest that however they were trained, pulmonologists and neurologists in Michigan show indistinguishable approaches to the diagnosis and treatment of SDB. The sample size and study design did not permit valid comparisons of approaches used by other specialists, but the results suggest a good degree of cross-fertilization between primary specialties involved in sleep medicine.

The rate that new, potential SDB patients were evaluated by each practitioner-at a median of 400 per year, or 8 per week-suggests that most sleep specialists spend much if not most of their time seeing patients with conditions other than SDB. As SDB constitutes nearly $70 \%$ of most sleep center practices, ${ }^{3}$ the majority of respondents' patients are probably seen for conditions other than sleep disorders. However, the wide range in the numbers of SDB patients seen by individual clinicians suggests that some are seeing mostly or only sleep disorder patients. Whether practice volume affects quality of care delivered cannot be assessed with current data.

Perhaps the most interesting finding of this study is the large degree to which a patient's experience may vary depending on the individual clinician seen. Split-night sleep studies for the diagnosis and treatment of SDB may save time and money spent in a sleep laboratory, but may not be as effective as separate full-night diagnostic and titration studies. Consensus recommendations suggest that split-night studies be performed only under specific circumstances, ${ }^{7}$ but they appear to be applied to $\sim 1$ in 4 patients evaluated for SDB in Michigan. The wide variation in the frequency of split-night studies in different practices-from 0 to $90 \%$ would be difficult to explain by widely different types of patient populations, and more likely reflects practitioner preferences or laboratory bed availability. Less variance was seen in the low frequency of ambulatory studies, but this finding may result from a lack of reimbursement for these procedures in Michigan.

Similarly, wide variance was seen in approaches to treatment of SDB. Prescriptions for CPAP and BPAP each were rare in some practices and exceedingly common in others; automatically titrating CPAP was used rarely for the most part but for $100 \%$ of treated patients in at least one practice. Surgery was offered to three times as many patients as oral appliance therapy, again with wide variance even among ABSM-certified (and therefore nonsurgical) state society members. Although uvulopalatopharyngoplasty was by far the most common inpatient surgery reported, in some practices large numbers of patients were treated with procedures unavailable at other sites. Some of the variance is likely to stem from differences in the nature of the patients seen: a practitioner who sees many children with craniofacial disorders or adults with morbid obesity might be more likely than another clinician to have his or her patients treated by tracheostomy or skeletal surgery. However, much of the difference likely reflects other factors about which patients may not be readily aware, including individual practitioner experience and availability of specific surgical specialists in local vicinities. For example, wide variance in frequency of positional therapy more likely reflects differences in attitudes or experience rather than resource availability, whereas variance in use of genioglossus advancement-from 0 to $30 \%$ of inpatient surgeries - more likely reflects both availability and attitudes of surgeons trained to perform the procedure. 
One limitation of this study is that only about two thirds of mailed surveys were returned, despite a follow-up request. This response rate is good for a survey aimed at physicians. ${ }^{8}$ In addition, comparison of initial respondents to those who participated only after the second mailing suggested no substantial differences. However, late participants may not fully reflect practices of nonrespondents. Furthermore, the membership of the Michigan Sleep Disorders Association probably omits a limited number of physicians who practice sleep medicine in this state. Therefore, some selection bias may have influenced median responses and limited generalizability. However, any such bias would have artificially limited, not magnified, the already large practice variations that form the main finding of this report.

Sleep medicine is a comparatively young field, and evidence-based, widely accepted, uniform strategies for many aspects of SDB assessment and management are not yet available. ${ }^{9}$ Standardization of options available to patients may not be desirable until optimal strategies have been more clearly identified. Aside from standard diagnosis with full-night polysomnography and treatment with nasal CPAP, many of the diagnostic and therapeutic variations in SDB management have not been studied rigorously in appropriately blinded, controlled, and randomized research. Until such data are available, clinicians should retain a broad degree of circumspection about their own practices in relation to others, and patients should be informed of all options and reasons behind clinicians' recommendations for specific strategies.

\section{ACKNOWLEDGMENTS}

The authors thank Harvey W. Organek, M.D., President, and Cindy Nichols, Ph.D., Secretary, Michigan Sleep Disorders Association, for their assistance with initial planning and implementation of this survey.

\section{REFERENCES}

1. Chervin RD, Murman DL, Malow BA. Totten V. Costutility of three approaches to the diagnosis of sleep apnea: polysomnography, home testing, and empirical therapy. Ann Intern Med 1999;130:496-505

2. Coleman RM, Roffwarg HP, Kennedy SJ, et al. Sleep-wake disorders based on a polysomnographic diagnosis. A national cooperative study. JAMA 1982;247:997-1003

3. Punjabi NM, Welch D, Strohl K. Sleep disorders in regional sleep centers: a national cooperative study. Coleman II Study Investigators. Sleep 2000;23:471-480

4. Dillman DA. Mail and Telephone Surveys: The Total Design Method. New York, NY: John Wiley; 1978

5. Young T, Palta M, Dempsey J, et al. The occurrence of sleepdisordered breathing among middle-aged adults. N Engl J Med 1993;328:1230-1235

6. Young T, Evans L, Finn L, Palta M. Estimation of the clinically diagnosed proportion of sleep apnea syndrome in middle-aged men and women. Sleep 1997;20:705-706

7. Chesson AL, Ferber RA, Fry JM, et al. The indications for polysomnography and related procedures. Sleep 1997;20: 423-487

8. Streiff MB, Dundes L, Spivak JL. A mail survey of United States hematologists and oncologists: a comparison of business reply versus stamped return envelopes. J Clin Epidemiol 2001;54:430-432

9. Boehlecke B. Controversies in monitoring and testing for sleep-disordered breathing. Curr Opin Pulm Med 2001;7: 372-380 